\title{
Asymmetric Hubbard model within generating functional approach in dynamical mean field theory
}

\author{
I.V.Stasyuk, O.B.Hera \\ Institute for Condensed Matter Physics of the National Academy of Sciences of Ukraine, \\ 1 Svientsitskii Str., 79011 Lviv, Ukraine
}

Received June 6, 2006

\begin{abstract}
In the paper a new analytic approach to the solution of the effective single-site problem in the dynamical mean field theory is developed. The approach is based on the method of the Kadanoff-Baym generating functional in the form developed by Izyumov et al. It makes it possible to obtain a closed equation in functional derivatives for the irreducible part of the single-site particle Green's function; the solution is constructed iteratively. As an application of the proposed approach the asymmetric Hubbard model (AHM) is considered. The inverse irreducible part $\Xi_{\sigma}^{-1}$ of the single-site Green's function is constructed in the linear approximation with respect to the coherent potential $J_{\sigma}$. Basing on the obtained result, the Green's function of itinerant particles in the Falicov-Kimball limit of AHM is considered, and the decoupling schemes in the equations of motion approach (GH3 approximation, decoupling by Jeschke and Kotliar) are analysed.
\end{abstract}

Key words: strongly correlated systems, asymmetric Hubbard model, single-site problem, dynamical mean field theory

PACS: $71.10 . F d, 71.27 .+a, 71.30 .+h$

\section{Introduction}

The lattice models with Hubbard correlations are used in investigating strongly-correlated materials. The one-band Hubbard model [1,2] and the spinless Falicov-Kimball model [3] can be considered as the simplest models of this kind. In the present paper those models are combined into the asymmetric Hubbard model describing a system with two sorts of mobile particles (electrons, ions, ...) with different hopping integrals and different values of chemical potentials. Thus, the model can be considered in investigating mixed-valence compounds [4] or ionic conductors. The model Hamiltonian has the following form:

$$
\begin{gathered}
\hat{H}=\sum_{i} \hat{H}_{i}+\sum_{i j \sigma} t_{i j}^{\sigma} a_{i \sigma}^{\dagger} a_{j \sigma}, \\
\hat{H}_{i}=U n_{i \uparrow} n_{i \downarrow}-\sum_{\sigma \in\{\downarrow, \uparrow\}} \mu_{\sigma} n_{i \sigma} .
\end{gathered}
$$

Here, the electron transfer is described using fermionic creation and annihilation operators $\left(a_{i \sigma}^{\dagger}\right.$, $\left.a_{j \sigma}\right)$ and hopping parameters $t_{i j}^{\sigma}$. The single site part $H_{i}$ contains the Coulomb repulsion $U\left(n_{i \sigma}=\right.$ $\left.a_{i \sigma}^{\dagger} a_{i \sigma}\right)$ and chemical potentials $\mu_{\sigma}$. If the model is used for describing two sorts of fermionic quasiparticles the electron spin indices are replaced by sort indices $\sigma=A, B$. The hopping integrals and chemical potentials in asymmetric Hubbard model are dependent on particle sort:

$$
t_{i j}^{A} \neq t_{i j}^{B}, \quad \mu_{A} \neq \mu_{B} .
$$

However, even such a relatively simple model cannot be solved exactly and various simplifications are required in investigating the problem. As for now, only some specific cases were considered. Thus, in the ground state of the model, a possibility of a phase separation phenomenon was analyzed in [5]. In the case of large on-site repulsion $U$, it is possible to use the effective anisotropic 
Heisenberg model with antiferromagnetic interaction [4,6,7]. Also, some thermodynamic properties of the model were investigated in the case of one-dimensional space $[7,8]$. In infinite dimensions, the spectral functions (densities of states) in the asymmetric Hubbard model were investigated using approximations in the equation of motion approach for Green's functions [9,10].

In this paper we consider the model within the dynamical mean field theory (DMFT) that is exact in the limit of infinite dimension. In this case the lattice model is reduced to the singlesite problem where the self energy $\Sigma(\mathbf{k})$ is independent of the wave vector $\mathbf{k}$ [11]. The single-site problem can be formulated constructing the effective Hamiltonian:

$$
\mathrm{e}^{-\beta H} \rightarrow \mathrm{e}^{-\beta H_{\text {eff }}}=\mathrm{e}^{-\beta H_{0}} \mathcal{T} \exp \left[-\int_{0}^{\beta} \mathrm{d} \tau \int_{0}^{\beta} \mathrm{d} \tau^{\prime} \sum_{\sigma} J_{\sigma}\left(\tau-\tau^{\prime}\right) a_{\sigma}^{\dagger}(\tau) a_{\sigma}\left(\tau^{\prime}\right)\right]
$$

where $H_{0}=\sum_{i} \hat{H}_{i}$. A solution of the single-site problem is supplemented by the solution of the Larkin equation

$$
G_{\sigma}\left(\omega_{n}\right)=\frac{1}{\Xi_{\sigma}^{-1}\left(\omega_{n}\right)-J_{\sigma}\left(\omega_{n}\right)}
$$

and the relation following from the equality of the lattice Green's function and the single-site Green's function:

$$
G_{\sigma}\left(\omega_{n}\right)=\int_{-\infty}^{+\infty} \frac{\rho_{\sigma}^{0}(t) \mathrm{d} t}{\Xi_{\sigma}^{-1}\left(\omega_{n}\right)-t},
$$

where $\rho_{\sigma}^{0}(t)$ is unperturbed particle density of states.

Let us note that for solving the single-site problem (3), it can be reformulated in terms of the single impurity Anderson model [12] or using the auxiliary Fermi-field describing the effective external bath as it was done in $[9,10,13,14]$.

The task of the single-site problem is to find the functional relation between the single-site Green's function $G_{\sigma}$ and the coherent potential $J_{\sigma}$. A number of numerical methods has been developed for this purpose (for example, the quantum Monte-Carlo [15-17], the exact diagonalization $[18,19]$, and the numerical renormalization group [20]). However, with the recent development of the $a b$ initio methods for calculating electronic structure of real materials combining the density functional theory with the dynamical mean field theory (see reviews $[21,22]$ ), new fast methods for solving the single-site problem are required. The analytic approximations for the Green's function (self-energy) in terms of the coherent potential can be used as such fast methods [13,23-27]. Among them there are methods based on decoupling of Green's functions in the equation of motion approach [13,23] and methods based on perturbation theory expansion (for example, iterated perturbation theory $[12,28]$ ). As shown in [13], the different-time decoupling approach includes the modified alloy-analogy approximation [29] and the Hubbard-III approximation [30] as particular cases.

In this paper, to systematize and consider a possibility of improving analytic methods we develop the generating functional approach as solver for the single-site problem. This method is based on the Kadanoff and Baym functional scheme [31] in the form elaborated by Izyumov and Chaschin for the lattice models with strong correlations (for example, Hubbard model and Heisenberg model) [32-35]. The approach allows one to obtain a closed equation in functional derivatives for irreducible part of the Green's function. Solutions of the equation are constructed iteratively in a form of expansion around the atomic limit (in powers of hopping $t_{i j}$ or $J_{\sigma}$ )

Exemplified by the asymmetric Hubbard model, it is shown that the problem can be formulated as the equation for the Larkin irreducible part $\Xi(\omega)$ or the equation for the self-energy and terminal part of the Green's function. This technique allows one to construct an analytic expression for the irreducible part with arbitrary precision in powers of hopping (coherent potential). The first iteration leads after some simplification to the so-called generalized Hubbard-III (GH3) approximation that was recently proposed using different-time decoupling of irreducible Green's functions in the equations of motion approach $[9,14]$. In the same way, there is established a relation with the decoupling scheme of Green's functions of higher order used by Jeschke and Kotliar [23] for the Hubbard model at $U \rightarrow \infty$ (JK decoupling). The generating functional approach enables us to improve these approximations constructing successive iterations for the irreducible part. 


\section{Effective single-site problem and generating functional approach}

Let us consider the effective single-site problem (3) using the procedure proposed in [13] where the problem was reformulated in terms of the auxiliary Fermi-field. In this case, the Hamiltonian $H_{\text {eff }}$ can be written explicitly

$$
H_{\mathrm{eff}}=H_{i}+\sum_{\sigma} V_{\sigma}\left(a_{\sigma}^{\dagger} \xi_{\sigma}+\xi_{\sigma}^{\dagger} a_{\sigma}\right)+H_{\xi}
$$

where the last term $\left(H_{\xi}\right)$ characterizes the environment of a given site in terms of $\xi$-field. The Green's function

$$
\mathcal{G}_{\sigma}\left(\tau-\tau^{\prime}\right)=\left\langle\mathcal{T}_{\tau} \xi_{\sigma}^{\dagger}(\tau) \xi_{\sigma}\left(\tau^{\prime}\right)\right\rangle_{0}
$$

that is calculated by formal averaging with the zero-order Hamiltonian $H_{\xi}$, determines the coherent potential

$$
J_{\sigma}\left(\tau-\tau^{\prime}\right)=V_{\sigma}^{2} \mathcal{G}_{\sigma}\left(\tau-\tau^{\prime}\right) \equiv V_{\sigma}^{2}\left\langle\mathcal{T}_{\tau} \xi_{\sigma}^{\dagger}(\tau) \xi_{\sigma}\left(\tau^{\prime}\right)\right\rangle_{0}
$$

The main task in the given approach is to calculate the single-site Green's function

$$
G_{\sigma}=\left\langle\mathcal{T}_{\tau} a_{\sigma}^{\dagger}(\tau) a_{\sigma}\left(\tau^{\prime}\right)\right\rangle
$$

for the problem with the Hamiltonian (6) and defined function $\mathcal{G}_{\sigma}$. As a result, such a solution gives the relation between the single-site Green's function $G_{\sigma}$ and coherent potential $J_{\sigma}$.

In the Hubbard operators representation the creation and annihilation operators are expressed as

$$
a_{\sigma}=X^{0 \sigma}+\zeta X^{\bar{\sigma} 2}, \quad a_{\sigma}^{\dagger}=X^{\sigma 0}+\zeta X^{2 \bar{\sigma}},
$$

where indices and signs are determined as follows

$$
\begin{cases}\bar{\sigma}=B, \zeta=+ & \text { for } \sigma=A, \\ \bar{\sigma}=A, \zeta=- & \text { for } \sigma=B .\end{cases}
$$

Then, the Hamiltonian of the effective single-site problem reads

$$
\begin{aligned}
H_{\mathrm{eff}} & =H_{0}+H_{\mathrm{int}}, \\
H_{0} & =U X^{22}-\sum_{\sigma}\left[\mu_{\sigma}\left(X^{\sigma \sigma}+X^{22}\right)\right]+H_{\xi}, \\
H_{\mathrm{int}} & =\sum_{\sigma} V_{\sigma}\left[\left(X^{\sigma 0}+\zeta X^{2 \bar{\sigma}}\right) \xi_{\sigma}+\xi_{\sigma}^{\dagger}\left(X^{0 \sigma}+\zeta X^{\bar{\sigma} 2}\right)\right] .
\end{aligned}
$$

We use below the thermodynamic perturbation theory. For this purpose the term that describes the hopping between the given site and environment in (11) is considered as a perturbation (interaction) term $H_{\text {int }}$. The scheme of calculating the Green's function

$$
\begin{aligned}
G_{\sigma}\left(\tau-\tau^{\prime}\right) \equiv & \left\langle T_{\tau} a_{\sigma}^{\dagger}(\tau) a_{\sigma}\left(\tau^{\prime}\right)\right\rangle=\left\langle T_{\tau} X^{\sigma 0}(\tau) X^{0 \sigma}\left(\tau^{\prime}\right)\right\rangle+\zeta\left\langle T_{\tau} X^{\sigma 0}(\tau) X^{\bar{\sigma} 2}\left(\tau^{\prime}\right)\right\rangle \\
& +\left\langle T_{\tau} X^{2 \bar{\sigma}}(\tau) X^{\bar{\sigma} 2}\left(\tau^{\prime}\right)\right\rangle+\zeta\left\langle T_{\tau} X^{2 \bar{\sigma}}(\tau) X^{0 \sigma}\left(\tau^{\prime}\right)\right\rangle
\end{aligned}
$$

is based on the Kadanoff and Baym generating functional method [31] applied and developed for the problems with strong particle correlations by Izyumov et al. [35]. To simplify the formulation of the method we consider the limit case $U \rightarrow \infty$, when the doubly occupied site is excluded, and the problem is reduced to the calculation of the Green's function $G^{\sigma 0,0 \sigma} \equiv\left\langle\mathcal{I}_{\tau} X^{\sigma 0}(\tau) X^{0 \sigma}\left(\tau^{\prime}\right)\right\rangle$.

Let us introduce the time dependent fluctuating fields $v_{\gamma}(\tau)$ that are conjugated to the quantities described by corresponding bosonic Hubbard operators $X^{\gamma}(\gamma=00, \sigma \sigma, \sigma \bar{\sigma})$. In that case the partition function is rewritten in the following form

$$
Z \rightarrow Z_{V}=\operatorname{Sp}\left(\mathrm{e}^{-\beta H} \mathcal{T}_{\tau} \mathrm{e}^{-V}\right)
$$


where

$$
V=\int_{0}^{\beta} \mathrm{d} \tau V(\tau)=\int_{0}^{\beta} \mathrm{d} \tau \sum_{\gamma} v_{\gamma}(\tau) X^{\gamma}(\tau)
$$

The calculation of operator average values (averaging over Gibbs ensemble with Hamiltonian $H_{\text {eff }}$ in the presence of fluctuating fields) is performed according to the formula:

$$
\langle\ldots\rangle_{V}=\frac{\operatorname{Sp}\left(\mathcal{I}_{\tau} \mathrm{e}^{-\beta H} \ldots \mathrm{e}^{-V}\right)}{\operatorname{Sp}\left(\mathcal{T}_{\tau} \mathrm{e}^{-\beta H} \mathrm{e}^{-V}\right)} .
$$

The average values of bosonic operators $X^{\gamma}$ are expressed as functional derivatives with respect to $v_{\gamma}$ :

$$
\left\langle X^{\gamma}\right\rangle=\left.\left\langle X^{\gamma}\right\rangle_{V}\right|_{V \rightarrow 0}=-\left.\frac{1}{Z_{V}} \frac{\delta Z}{\delta v_{i}(\tau)}\right|_{V \rightarrow 0},
$$

where the fields $v_{\gamma}$ are directed to zero after the differentiation to obtain the operator average values for the system with initial Hamiltonian.

Introducing the generating functional [35]

$$
Z_{V}=\mathrm{e}^{\Phi_{V}}, \quad \Phi_{V}=\ln Z_{V},
$$

we can rewrite the relation (18) in the following form

$$
\left\langle X^{\gamma}\right\rangle=-\left.\frac{\delta \Phi_{V}}{\delta v_{i}(\tau)}\right|_{V \rightarrow 0} .
$$

From the expression for the second-order derivatives

$$
\frac{\delta^{2} \Phi_{V}}{\delta v_{\alpha}\left(\tau_{1}\right) \delta v_{\beta}\left(\tau_{2}\right)}=\frac{1}{Z_{V}} \frac{\delta^{2} Z_{V}}{\delta v_{\alpha}\left(\tau_{1}\right) \delta v_{\beta}\left(\tau_{2}\right)}-\frac{1}{Z_{V}^{2}} \frac{\delta Z_{V}}{\delta v_{\alpha}\left(\tau_{1}\right)} \frac{\delta Z_{V}}{\delta v_{\beta}\left(\tau_{2}\right)}
$$

(and the similar ones for the higher-order derivatives) follows a relation between functional derivatives of average values of bosonic $X$-operators (Green's functions) and the higher order Green's functions on such operators:

$$
\frac{\delta}{\delta v_{\beta}\left(\tau_{2}\right)}\left\langle\mathcal{T}_{\tau} X^{\alpha}\left(\tau_{1}\right)\right\rangle_{V}=-\left\langle\mathcal{T}_{\tau} X^{\alpha}\left(\tau_{1}\right) X^{\beta}\left(\tau_{2}\right)\right\rangle_{V}+\left\langle\mathcal{T}_{\tau} X^{\alpha}\left(\tau_{1}\right)\right\rangle_{V}\left\langle\mathcal{I}_{\tau} X^{\beta}\left(\tau_{2}\right)\right\rangle_{V} .
$$

Similarly, one can write an arbitrary Green's function of the higher order with bosonic Hubbard operator using functional derivatives:

$$
\begin{aligned}
\left\langle\mathcal{T}_{\tau} X^{\alpha}\left(\tau_{1}\right) \ldots\right\rangle_{V} & =-\frac{\delta\left\langle\mathcal{T}_{\tau} \ldots\right\rangle_{V}}{\delta v_{\alpha}\left(\tau_{1}\right)}-\left\langle\mathcal{T}_{\tau} \ldots\right\rangle_{V} \frac{\delta \Phi_{V}}{\delta v_{\alpha}\left(\tau_{1}\right)} \\
& =-\frac{\delta\left\langle\mathcal{T}_{\tau} \ldots\right\rangle_{V}}{\delta v_{\alpha}\left(\tau_{1}\right)}+\left\langle\mathcal{T}_{\tau} \ldots\right\rangle_{V}\left\langle\mathcal{T}_{\tau} X^{\alpha}\left(\tau_{1}\right)\right\rangle_{V} .
\end{aligned}
$$

The last relation makes it possible to obtain a closed set of equations in functional derivatives for the fermionic Green's functions

$$
G_{V}^{\sigma 0,0 \eta}\left(\tau, \tau^{\prime}\right) \equiv\left\langle\mathcal{T}_{\tau} X^{\sigma 0}(\tau) X^{0 \eta}\left(\tau^{\prime}\right)\right\rangle_{V}
$$

The obtained solutions give the single-site Green's function $G^{\sigma 0,0 \sigma}\left(\tau-\tau^{\prime}\right)$ after the transition to the $v_{\gamma}=0$ limit. 


\subsection{Recurrent form of Wick's theorem}

Let us consider the procedure of constructing the equations for the Green's function with $X$ operators within the framework of thermodynamic perturbation theory. In this case

$$
Z_{V}=\operatorname{Sp}\left(\mathcal{T}_{\tau} \hat{\sigma}(\beta) \mathrm{e}^{-V} \mathrm{e}^{-\beta H_{0}}\right)=Z_{0}\left\langle\hat{\sigma}(\beta) \mathrm{e}^{-V}\right\rangle_{0},
$$

where

$$
\hat{\sigma}(\beta)=\mathcal{T}_{\tau} \exp \left[-\int_{0}^{\beta} \mathrm{d} \tau_{1} H_{\text {int }}\left(\tau_{1}\right)\right],
$$

and $Z_{0}=\mathrm{Spe}^{\beta H_{0}}$ is the zero-order partition function. Here, when $U \rightarrow \infty$, the zero-order Hamiltonian reads

$$
H_{0}=-\mu_{A} X^{A A}-\mu_{B} X^{B B}+H_{\xi}
$$

and the interaction (perturbation) term has the form

$$
H_{\mathrm{int}}=V_{A}\left(X^{A 0} \xi_{A}+\xi_{A}^{\dagger} X^{0 A}\right)+V_{B}\left(X^{B 0} \xi_{B}+\xi_{B}^{\dagger} X^{0 B}\right)
$$

Time ordered $\left(\mathcal{T}_{\tau}\right)$ operator averaging can be performed by calculating Gibbs average values with unperturbed Hamiltonian $H_{0}$

$$
\left\langle\mathcal{I}_{\tau} \ldots\right\rangle_{V}=\frac{\left\langle\mathcal{T}_{\tau} \ldots \hat{\sigma}(\beta) \mathrm{e}^{-V}\right\rangle_{0}}{\left\langle\mathcal{T}_{\tau} \hat{\sigma}(\beta) \mathrm{e}^{-V}\right\rangle_{0}}=\frac{Z_{0}}{Z_{V}}\left\langle\mathcal{I}_{\tau} \ldots \hat{\sigma}(\beta) \mathrm{e}^{-V}\right\rangle_{0}
$$

To calculate average values of time ordered $\left(\mathcal{I}_{\tau}\right)$ products of $X$-operators in $(29)$, we use a form of the Wick's theorem formulated in [36], that can be called its recurrent form. Let us write, in a closed form, the result of the pairing of one selected non-diagonal $X$-operator with all others

$$
\begin{aligned}
& \left\langle\mathcal{T}_{\tau} \underline{X}^{\sigma 0}(\tau) X^{\gamma_{1}}\left(\tau_{1}\right) \ldots X^{\gamma_{n}}\left(\tau_{n}\right)\right\rangle=\frac{Z_{0}}{Z_{V}} \sum_{i}\left\langle\underline{\mathcal{T}_{\tau} \underline{X^{\sigma 0}(\tau)} X^{\gamma_{1}}\left(\tau_{1}\right) \ldots \underline{X^{\gamma_{i}}}\left(\tau_{i}\right)} \ldots X^{\gamma_{n}}\left(\tau_{n}\right) \hat{\sigma}(\beta) \mathrm{e}^{-\hat{V}}\right\rangle_{0}
\end{aligned}
$$

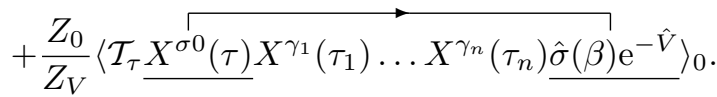

According to [36], the result of pairing of two selected operators is a product of their commutator (anticommutator, when both operators are of the Fermi-type) and the unperturbed Green's function. In that case

$$
\begin{aligned}
\left\langle\mathcal{T}_{\tau} X^{\sigma 0}(\tau)\right. & \left.X^{\gamma_{1}}\left(\tau_{1}\right) \ldots X^{\gamma_{n}}\left(\tau_{n}\right)\right\rangle=\frac{Z_{0}}{Z_{V}} \sum_{i} g^{\sigma 0}\left(\tau-\tau_{i}\right) \\
& \times\left\langle\mathcal{T}_{\tau} X^{\gamma_{1}}\left(\tau_{1}\right) \ldots\left[X^{\sigma 0}, X^{\gamma_{i}}\right]_{\tau_{i}}^{ \pm} \ldots X^{\gamma_{n}}\left(\tau_{n}\right) \hat{\sigma}(\beta) \mathrm{e}^{-\hat{V}}\right\rangle_{0}(-1)^{p_{i}} \\
& +\frac{Z_{0}}{Z_{V}} \int_{0}^{\beta} \mathrm{d} \tau^{\prime} g^{\sigma 0}\left(\tau-\tau^{\prime}\right)\left\langle\mathcal{T}_{\tau} X^{\gamma_{1}}\left(\tau_{1}\right) \ldots X^{\gamma_{n}}\left(\tau_{n}\right)\left[X^{\sigma 0}, H_{\mathrm{int}}+\hat{V}\right]_{\tau^{\prime}} \hat{\sigma}(\beta) \mathrm{e}^{-\hat{V}}\right\rangle_{0}
\end{aligned}
$$

where the alternating $(-1)^{p_{i}}$ multiplier is defined by a number of Fermi-permutations $p_{i}$ of operator $X^{\sigma 0}(\tau)$ from the starting position to the position directly on the left of the operator that is paired to.

Going back in (31) to the averages with the full (perturbed) Hamiltonian, we have

$$
\begin{gathered}
\left\langle\mathcal{T}_{\tau} X^{\sigma 0}(\tau) X^{\gamma_{1}}\left(\tau_{1}\right) \ldots X^{\gamma_{n}}\left(\tau_{n}\right)\right\rangle=\sum_{i} g^{\sigma 0}\left(\tau-\tau_{i}\right)\left\langle\mathcal{T}_{\tau} X^{\gamma_{1}}\left(\tau_{1}\right) \ldots\left[X^{\sigma 0}, X^{\gamma_{i}}\right]_{\tau_{i}}^{ \pm} \ldots X^{\gamma_{n}}\left(\tau_{n}\right)\right\rangle(-1)^{p_{i}} \\
+\int_{0}^{\beta} \mathrm{d} \tau^{\prime} g^{\sigma 0}\left(\tau-\tau^{\prime}\right)\left\langle\mathcal{T}_{\tau} X^{\gamma_{1}}\left(\tau_{1}\right) \ldots X^{\gamma_{n}}\left(\tau_{n}\right)\left[X^{\sigma 0}, H_{\mathrm{int}}+\hat{V}\right]_{\tau^{\prime}}\right\rangle
\end{gathered}
$$


The similar procedure can be applied to the averages (Green's functions) involving $\xi$-operators. If the pairing procedure is started from the operator $\xi_{\sigma}^{\dagger}$, then, instead of unperturbed Green's function $g^{\sigma 0}\left(\tau-\tau^{\prime}\right)$, the Green's function of auxiliary fermions $\mathcal{G}_{\sigma}\left(\tau-\tau^{\prime}\right)$ is used and the $\xi_{\sigma}^{\dagger}$ operator is on the first position in the commutator.

Relation (32) can be called a recurrent form of the Wick's theorem. When it is used repeatedly to the averages appearing from the right hand side, the complete structure of the expansion of initial averages in products of unperturbed Green's functions is reproduced. However, the distinction is that the final expressions containing averages of diagonal $X$-operators that terminate the pairing sequence are calculated with the full Hamiltonian $H_{\text {eff }}$. It is different from using the standard Wick's theorem [36] where such averages are taken over the Gibbs ensemble with the unperturbed Hamiltonian $H_{0}$.

Let us note, that the formula (32) can be interpreted as an equation relating the averages (Green's functions) with the Green's functions of higher order. The same structure of equations is characteristic of the equation of motion approach [31] for the temperature Matsubara Green's functions.

\subsection{Equations for single-particle Green's function}

The relation (32) is applied to the Green's function $\left\langle\mathcal{T}_{\tau} X^{\sigma 0}(\tau) X^{0 \eta}\left(\tau^{\prime}\right)\right\rangle_{V}$. For this purpose, we need to calculate the commutators of the Hubbard operator $X^{\sigma 0}$ with $H_{\text {int }}+V$ and anticommutator of operators of Fermi-type $X^{\sigma 0}, X^{0 \eta}$. For example:

$$
\begin{aligned}
{\left[X^{B 0}, H_{\mathrm{int}}\right] } & =-V_{A} \xi_{A}^{\dagger} X^{B A}-V_{B} \xi_{B}^{\dagger}\left(X^{00}+X^{B B}\right), \\
{\left[X^{B 0}, V\right] } & =-\left(v_{B}-v_{0}\right) X^{B 0}-\bar{v} X^{A 0}, \\
\left\{X^{A 0}, X^{0 A}\right\} & =X^{00}+X^{A A}, \quad\left\{X^{A 0}, X^{0 B}\right\}=X^{A B},
\end{aligned}
$$

(where $v_{0} \equiv v_{00} ; v_{\sigma} \equiv v_{\sigma \sigma} ; \bar{v} \equiv v_{A B} ; v \equiv v_{B A}$ ). This leads to the appearance of Green's functions such as $\left\langle\mathcal{T}_{\tau} \xi_{\sigma}^{\dagger}\left(\tau_{1}\right)\left[X^{00}+X^{\sigma \sigma}\right]\left(\tau_{1}\right) X^{0 \eta}\left(\tau^{\prime}\right)\right\rangle_{V}$ and $\left\langle\mathcal{T}_{\tau} \xi_{\bar{\sigma}}^{\dagger}\left(\tau_{1}\right) X^{\sigma \bar{\sigma}}\left(\tau_{1}\right) X^{0 \eta}\left(\tau^{\prime}\right)\right\rangle_{V}$, which can be expressed using a relation like (32). Taking into account that

$$
\left[\xi_{A}^{\dagger}, H_{\mathrm{int}}\right]=-V_{A} X^{A 0}, \quad\left[\xi_{B}^{\dagger}, H_{\mathrm{int}}\right]=-V_{B} X^{B 0},
$$

we come to Green's functions with three Hubbard operators, where one operator is of a bosonic type. The Bose-operators are excluded using the functional differentiation (23). As a result we obtained the following equation for the Green's function $G_{V}^{\sigma 0,0 \eta}\left(\tau, \tau^{\prime}\right)$ :

$$
\begin{aligned}
G_{V}^{\sigma 0,0 \eta}\left(\tau, \tau^{\prime}\right)=-g_{\sigma 0}\left(\tau-\tau^{\prime}\right)\left[\left(\frac{\delta \Phi_{V}}{\delta v_{0}\left(\tau^{\prime}\right)}+\frac{\delta \Phi_{V}}{\delta v_{\sigma}\left(\tau^{\prime}\right)}\right) \delta_{\sigma, \eta}+\frac{\delta \Phi_{V}}{\delta \bar{v}_{\sigma, \bar{\sigma}}\left(\tau^{\prime}\right)} \delta_{\bar{\sigma}, \eta}\right]-\int_{0}^{\beta} \mathrm{d} \tau_{1} g_{\sigma 0}\left(\tau-\tau_{1}\right) \\
\quad \times \int_{0}^{\beta} \mathrm{d} \tau_{2} V_{\bar{\sigma}}^{2} \mathcal{G}_{\bar{\sigma}}\left(\tau_{1}-\tau_{2}\right)\left[\frac{\delta \Phi_{V}}{\delta \bar{v}_{\sigma, \bar{\sigma}}\left(\tau_{1}\right)} G_{V}^{\bar{\sigma} 0,0 \eta}\left(\tau_{2}, \tau^{\prime}\right)+\frac{\delta}{\delta \bar{v}_{\sigma, \bar{\sigma}}\left(\tau_{1}\right)} G_{V}^{\bar{\sigma} 0,0 \eta}\left(\tau_{2}, \tau^{\prime}\right)\right] \\
\quad-\int_{0}^{\beta} \mathrm{d} \tau_{1} g_{\sigma 0}\left(\tau-\tau_{1}\right) \int_{0}^{\beta} \mathrm{d} \tau_{2} V_{\sigma}^{2} \mathcal{G}_{\sigma}\left(\tau_{1}-\tau_{2}\right) \\
\quad \times\left[\left(\frac{\delta \Phi_{V}}{\delta v_{0}\left(\tau_{1}\right)}+\frac{\delta \Phi_{V}}{\delta v_{\sigma}\left(\tau_{1}\right)}+\frac{\delta}{\delta v_{0}\left(\tau_{1}\right)}+\frac{\delta}{\delta v_{\sigma}\left(\tau_{1}\right)}\right) G_{V}^{\sigma 0,0 \eta}\left(\tau_{2}, \tau^{\prime}\right)\right] \\
\quad+\int_{0}^{\beta} \mathrm{d} \tau_{1} g_{\sigma 0}\left(\tau-\tau_{1}\right)\left[v_{\sigma}\left(\tau_{1}\right)-v_{0}\left(\tau_{1}\right)\right] G_{V}^{\sigma 0,0 \eta}\left(\tau_{1}, \tau^{\prime}\right)+\int_{0}^{\beta} \mathrm{d} \tau_{1} g_{\sigma 0}\left(\tau-\tau_{1}\right) \bar{v}_{\bar{\sigma}, \sigma}\left(\tau_{1}\right) G_{V}^{\bar{\sigma} 0,0 \eta}\left(\tau_{1}, \tau^{\prime}\right),
\end{aligned}
$$

where $\delta_{\sigma, \eta}$ is a Kronecker delta and $\bar{v}_{B, A} \equiv v, \bar{v}_{A, B} \equiv \bar{v}$. 
Let us introduce an inverse Green's function $g_{\sigma 0}^{-1}\left(\tau-\tau^{\prime}\right)$ according to the definition

$$
\int_{0}^{\beta} \mathrm{d} \tau^{\prime} g_{\sigma 0}^{-1}\left(\tau-\tau^{\prime}\right) g_{\sigma 0}\left(\tau^{\prime}-\tau^{\prime \prime}\right)=\delta\left(\tau-\tau^{\prime \prime}\right)
$$

Accordingly, in frequency representation, it corresponds to

$$
g_{\sigma 0}^{-1}\left(\omega_{n}\right)=\left[g_{\sigma 0}\left(\omega_{n}\right)\right]^{-1}=\mathrm{i} \omega_{n}+\mu_{\sigma} .
$$

Let us multiply both sides of equation (34) by $g_{\sigma 0}^{-1}\left(\tau^{\prime \prime}-\tau\right)$ and integrate out the free time argument $\tau$. Then, we rewrite the obtained equation in more compact form using matrix representation where the required single-site Green's function has the following form

$$
\hat{G}=\left(\begin{array}{ll}
G^{B 0,0 B} & G^{B 0,0 A} \\
G^{A 0,0 B} & G^{A 0,0 A}
\end{array}\right) .
$$

Introducing a matrix representation for the differentiation operator

$$
\hat{A}^{\prime}\left(\tau, \tau^{\prime}\right)=\delta\left(\tau-\tau^{\prime}\right)\left(\begin{array}{ll}
\frac{\delta}{\delta v_{0 B}(\tau)} & \frac{\delta}{\delta v(\tau)} \\
\frac{\delta}{\delta \bar{v}(\tau)} & \frac{\delta}{\delta v_{0 A}(\tau)}
\end{array}\right),
$$

where $\frac{\delta}{\delta v_{0 \sigma}}=\frac{\delta}{\delta v_{0}}+\frac{\delta}{\delta v_{\sigma}}$, and introducing matrices for the Green's functions of auxiliary fermions (coherent potential)

$$
\hat{J}\left(\tau, \tau^{\prime}\right)=\left(\begin{array}{ll}
V_{B}^{2} \mathcal{G}_{B}\left(\tau-\tau^{\prime}\right) & 0 \\
0 & V_{A}^{2} \mathcal{G}_{A}\left(\tau-\tau^{\prime}\right)
\end{array}\right)=\left(\begin{array}{ll}
J_{B}\left(\tau-\tau^{\prime}\right) & 0 \\
0 & J_{A}\left(\tau-\tau^{\prime}\right)
\end{array}\right)
$$

and the inverse unperturbed Green's functions (combined with the linear contributions of the fluctuating field $V$ ):

$$
\hat{g}^{-1}\left(\tau, \tau^{\prime}\right)=\left(\begin{array}{cc}
g_{B 0}^{-1}\left(\tau-\tau^{\prime}\right) & 0 \\
0 & g_{A 0}^{-1}\left(\tau-\tau^{\prime}\right)
\end{array}\right)+\left(\begin{array}{cc}
v_{0}(\tau)-v_{B}(\tau) & \bar{v}(\tau) \\
-v(\tau) & v_{0}(\tau)-v_{A}(\tau)
\end{array}\right) \delta\left(\tau-\tau^{\prime}\right)
$$

the equation (34) can be presented in the form

$$
\hat{g}^{-1} \hat{G}+\hat{A}_{0} \hat{J} \hat{G}+\hat{A}^{\prime} \hat{J} \hat{G}=-\hat{A}_{0} .
$$

Here, the matrix $\hat{A}_{0}$ is a result of the differentiation operator $\hat{A}^{\prime}$ action on the generating functional $\Phi_{V}\left(\hat{A}_{0}=\hat{A}^{\prime} \Phi_{V}\right)$. The multiplication of arbitrary two matrices $\hat{X}\left(\tau, \tau^{\prime}\right)$ and $\hat{Y}\left(\tau^{\prime}, \tau^{\prime \prime}\right)$ contains integrating out the inner time argument $\tau^{\prime}$ :

$$
\hat{X} \hat{Y} \equiv \int_{0}^{\beta} \mathrm{d} \tau^{\prime} \hat{X}\left(\tau, \tau^{\prime}\right) \hat{Y}\left(\tau^{\prime}, \tau^{\prime \prime}\right) .
$$

At the final stage (after exclusion of fluctuating fields $v_{\gamma}(\tau)$ ), multiplication (42) corresponds to the product of Fourier's components in the frequency representation.

\subsection{Irreducible part}

We introduce the irreducible part $\hat{\Xi}$ of the single-site Green's function $\hat{G}$ meaning Larkin irreducibility. Then, the equation (4) can be written in the following form

$$
\hat{G}\left(\omega_{n}\right)=\left[\hat{\Xi}^{-1}\left(\omega_{n}\right)-\hat{J}\left(\omega_{n}\right)\right]^{-1} .
$$


To obtain an equation defining $\hat{\Xi}$ we use a property of the differentiation

$$
\frac{\delta}{\delta v} \hat{G}=-\hat{G}\left[\frac{\delta}{\delta v} \hat{G}^{-1}\right] \hat{G},
$$

and rewrite the equation (41) in the form of a functional differential equation where the differentiation operator $\hat{A}^{\prime}$ acts on the inverse Green's function matrix $G^{-1}$ :

$$
\hat{G}^{-1}=-\hat{A}_{0}^{-1} \hat{g}^{-1}-\hat{J}-\hat{A}_{0}^{-1} \overrightarrow{\hat{A}^{\prime}} \hat{\jmath} \hat{G} \hat{G}^{-1} \hat{G} \hat{G}^{-1}
$$

the arrow line points a matrix function operated on by the differentiation operators. Transforming this equation we obtain the equation for the irreducible part:

$$
\hat{\Xi}^{-1}=\hat{G}_{0}^{-1}+\hat{A}_{0}^{-1} \overrightarrow{\hat{A}^{\prime}} \hat{J} \hat{G}^{-} \hat{\Xi}^{-1}
$$

where

$$
G_{0}^{-1}=-\hat{A}_{0}^{-1} \hat{g}^{-1}
$$

In explicit form it reads

$$
\begin{aligned}
\left(\hat{\Xi}^{-1}\right)_{\mathrm{pl}}\left(\tau, \tau^{\prime}\right)= & \left(\hat{G}_{0}^{-1}\right)_{\mathrm{pl}}\left(\tau, \tau^{\prime}\right)+\sum_{s q m} \int_{0}^{\beta} \mathrm{d} \tau_{1} \ldots \int_{0}^{\beta} \mathrm{d} \tau_{4} \hat{J}_{q q}\left(\tau_{1}, \tau_{4}\right) \hat{G}_{q m}\left(\tau_{4}, \tau_{2}\right)\left(\hat{A}_{0}^{-1}\right)_{p s}\left(\tau, \tau_{3}\right) \\
& \times\left[\hat{A}_{s q}^{\prime}\left(\tau_{3}, \tau_{1}\right)\left(\hat{\Xi}^{-1}\right)_{m l}\left(\tau_{2}, \tau^{\prime}\right)\right], \\
\hat{G}_{0}^{-1}\left(\tau, \tau^{\prime}\right)=- & \frac{1}{D(\tau)}\left(\begin{array}{cc}
\frac{\delta \Phi_{V}}{\delta v_{0 A}(\tau)} g_{B 0}^{-1}\left(\tau-\tau^{\prime}\right) & -\frac{\delta \Phi_{V}}{\delta v(\tau)} g_{A 0}^{-1}\left(\tau-\tau^{\prime}\right) \\
-\frac{\delta \Phi_{V}}{\delta \bar{v}(\tau)} g_{B 0}^{-1}\left(\tau-\tau^{\prime}\right) & \frac{\delta \Phi_{V}}{\delta v_{0 B}(\tau)} g_{A 0}^{-1}\left(\tau-\tau^{\prime}\right)
\end{array}\right) \\
& -\left(\begin{array}{ll}
\frac{\delta \Phi_{V}}{\delta v_{0 A}(\tau)}\left[v_{0}(\tau)-v_{B}(\tau)\right]+\frac{\delta \Phi_{V}}{\delta v(\tau)} v(\tau) & -\frac{\delta \Phi_{V}}{\delta v_{0 A}(\tau)} \bar{v}(\tau)-\frac{\delta \Phi_{V}}{\delta v(\tau)}\left[v_{0}(\tau)-v_{A}(\tau)\right] \\
-\frac{\delta \Phi_{V}}{\delta \bar{v}(\tau)}\left[v_{0}(\tau)-v_{B}(\tau)\right]-\frac{\delta \Phi_{V}}{\delta v_{0 B}(\tau)} v(\tau) & \frac{\delta \Phi_{V}}{\delta \bar{v}(\tau)} \bar{v}(\tau)+\frac{\delta \Phi_{V}}{\delta v_{0 B}(\tau)}\left[v_{0}(\tau)-v_{A}(\tau)\right]
\end{array}\right) \\
& \times \frac{1}{D(\tau)} \delta\left(\tau-\tau^{\prime}\right),
\end{aligned}
$$

where

$$
D(\tau)=\frac{\delta \Phi_{V}}{\delta v_{0 B}(\tau)} \frac{\delta \Phi_{V}}{\delta v_{0 A}(\tau)}-\frac{\delta \Phi_{V}}{\delta v(\tau)} \frac{\delta \Phi_{V}}{\delta \bar{v}(\tau)}
$$

Equation (46) has the structure that corresponds in the generating functional approach to the similar equations for irreducible parts of Green's functions for models of magnetics or strongly correlated electron systems [32-35]. Its solution can be sought in the form of a series with summands involving various number of $\hat{J}$ multipliers.

The iterations generate terms where operators $\hat{A}^{\prime}$ from (46) act only on matrices $\hat{A}_{0}^{-1}$ or $\hat{G}$ and do not affect the terminating matrix $\hat{g}^{-1}$ ( $\hat{G}_{0}^{-1}$ is taken as a zero order function). Another set is formed by summands involving derivatives of $\hat{g}^{-1}$. Therefore, the solution can be presented in the form

$$
\hat{\Xi}^{-1}=-\hat{A}_{0}^{-1} \hat{M}\left(\hat{g}^{-1}+\hat{F}\right)
$$


where two new matrices are introduced $(\hat{M}$ and $\hat{F})$, and the mentioned terms with differentiation of $\hat{g}^{-1}$ are collected in $\hat{F}$.

Let us insert (51) into the equation (46). Collecting separately terms of the first and second type, we obtain two equations:

$$
\begin{gathered}
\hat{M}=\hat{1}+\overrightarrow{\hat{R} \hat{A}_{0}^{-1}} \hat{M}+\overrightarrow{\hat{R} \hat{A}_{0}^{-1} \hat{M}}, \\
\hat{F}=\hat{\hat{R}} \hat{A}_{0}^{-1} \hat{M}^{-1}+\hat{\hat{R}} \hat{A}_{0}^{-1} \hat{M} \hat{F}
\end{gathered}
$$

where the notation

$$
\overrightarrow{\hat{R} \cdots}=\overrightarrow{\hat{A}^{\prime} \hat{J} \hat{G} \ldots}
$$

is introduced.

Iterations in the equation (52) point to a possibility of writing the matrix $\hat{M}$ as

$$
\hat{M}=(1-\hat{L})^{-1},
$$

where the matrix $\hat{L}$ is determined by the functional equation

$$
\hat{L}=\hat{L}_{0}+\hat{R} \hat{A}_{0}^{-1}[\hat{1}-\hat{L}]^{-1} \hat{L}
$$

where

$$
\hat{L}_{0}=\overrightarrow{\hat{R}} \hat{A}_{0}^{-1} \equiv \overrightarrow{A^{\prime} J G A_{0}^{-1}}
$$

Accordingly, the equation (53) is rewritten as

$$
\hat{F}=\hat{F}_{0}+\hat{R} \hat{A}_{0}^{-1}[\hat{1}-\hat{L}]^{-1} \hat{F}
$$

where

$$
F_{0}=\hat{R} \hat{A}_{0}^{-1}[\hat{1}-\hat{L}]^{-1} \hat{g}^{-1} .
$$

Let us note, that matrix $\hat{M}$ can be connected with the so-called terminating part $\hat{\Lambda}$ of the Green's function $\hat{G}$. Let us write $\hat{G}$ in the form

$$
\hat{G}=\hat{\Pi} \hat{\Lambda},
$$

and introduce the mass operator $\hat{\Sigma}$ according to the equation

$$
\hat{\Pi}=\hat{g}+\hat{g} \hat{\Sigma} \hat{\Pi}, \quad \hat{\Pi}^{-1}=\hat{g}^{-1}-\hat{\Sigma} .
$$

In this case the equation for the Green's function $\hat{G}$ (41) can be split into two equations for the mass operator $\hat{\Sigma}$ and terminating part $\hat{\Lambda}$, as it was done by Izyumov et al. [32-35]:

$$
\begin{gathered}
\hat{\Sigma}+\hat{A}_{0} \hat{J}-\overrightarrow{\hat{A}^{\prime} \hat{J} \hat{\Pi} \hat{g}^{-1}}+\overrightarrow{\hat{A}^{\prime} \hat{J} \hat{\Pi} \hat{\Sigma}}=0, \\
\hat{\Lambda}+\overrightarrow{\hat{A}^{\prime} \hat{J} \hat{\Pi} \hat{\Lambda}}=-A_{0} .
\end{gathered}
$$

Consider the relation between the mass operator $\hat{\Sigma}$ and irreducible part $\hat{\Xi}$

$$
\hat{\Sigma}=\hat{g}^{-1}+\hat{\Lambda} \hat{J}-\hat{\Lambda} \hat{\Xi}^{-1},
$$


following from the relations (60), (61) and equation (46). Comparing equations (56), (58) and (62), (63), one can see that there exists the correspondence

$$
\begin{gathered}
\hat{R} \hat{A}_{0}^{-1}[\hat{1}-\hat{L}]^{-1} \cdots=-\overrightarrow{\hat{A}^{\prime} \hat{J} \hat{\Pi} \ldots} \\
\hat{\Lambda}=-[\hat{1}-\hat{L}] \hat{A}_{0}, \quad \hat{\Sigma}=\hat{\Lambda} \hat{J}-\hat{F} .
\end{gathered}
$$

This makes possible to write the inverse irreducible part of the Green's function as

$$
\hat{\Xi}^{-1}=\hat{\Lambda}^{-1}\left(\hat{g}^{-1}+\hat{F}\right) \text {. }
$$

From here, it is seen that $\hat{\Lambda}$ is also a terminating part of the matrix $\hat{\Xi}$, and $(-\hat{F})$ has the meaning of a Dyson irreducible part of $\hat{\Xi}$.

Summarizing, let us note that the equations (46) or (56) and (58) constructed by us allow one to find, respectively, the inverse irreducible part $\hat{\Xi}^{-1}$ or, separately, its components $\hat{L}$ and $\hat{F}$. Direct iteration procedure permits to construct their solutions in the form of functionals of $\hat{G}$ functions, bosonic correlators and coherent potential $\hat{J}$.

\section{Falicov-Kimball model: Green's function of itinerant particles}

As an example of the simple application, we consider within the framework of the developed approach the Green's function of itinerant particles in the Falicov-Kimball model as the simplest specific case. This problem can be exactly solved in DMFT and its solution corresponds to the so-called alloy-analogy (AA) approach.

In the case of the Falicov-Kimball model, particles of one sort are localized $\left(J_{B}=0\right.$; in the matrix notations $\hat{J}_{11}=0$ ). To find the Green's function of itinerant particles (corresponding matrix element $\left.(\hat{G})_{22}\right)$ the irreducible part $\left(\hat{\Xi}^{-1}\right)_{22}$ should be calculated.

The series for $\left(\hat{\Xi}^{-1}\right)_{22}$ does not contain nonzero terms where the differentiation operator $\hat{A}^{\prime}$ acts on the zero-order Green's function $\hat{g}$ :

$$
\left(\hat{F}_{1}\right)_{22}=0, \quad\left(\hat{F}_{2}\right)_{22}=0,
$$

because

$$
\left(\hat{A}^{\prime}\right)_{22}\left(\hat{g}^{-1}\right)_{22}=0,
$$

and, in terms where the operator $\left(\hat{A}^{\prime}\right)_{12}$ acts on $\left(\hat{g}^{-1}\right)_{12}$, either matrix element $\left(\hat{A}_{0}^{-1}\right)_{21}$ or $\hat{G}_{21}$ is present that gives a vanishing result when the fluctuating field tends to zero. Thus

$$
\left(\hat{\Xi}^{-1}\right)_{22}=-\left(\hat{A}_{0}^{-1}\right)_{22}\left([\hat{1}-\hat{L}]^{-1}\right)_{22}\left(g_{A 0}^{-1}\right),
$$

and taking into account that when $v \rightarrow 0$, the matrix $\hat{L}$ is diagonal:

$$
\left(\hat{\Xi}^{-1}\right)_{22}=-\left(\hat{A}_{0}^{-1}\right)_{22}\left[1-\hat{L}_{22}\right]^{-1} g_{A 0}^{-1} .
$$

To find the function $\hat{L}$, we use a fact that operator $X^{00}+X^{A A}$ is an integral of motion in the case $J_{B}=0\left(\left[X^{00}+X^{A A}, H_{\text {eff }}+V\right]=0\right)$. It gives

$$
\begin{aligned}
\left\langle\mathcal{T}_{\tau}\left(X^{00}+X^{A A}\right)(\tau)\left(X^{00}+X^{A A}\right)\left(\tau^{\prime}\right)\right\rangle & =\left\langle X^{00}+X^{A A}\right\rangle, \\
\left\langle\mathcal{I}_{\tau}\left(X^{00}+X^{A A}\right)\left(\tau_{1}\right) X^{A 0}(\tau) X^{0 A}\left(\tau^{\prime}\right)\right\rangle & =\left\langle\mathcal{T}_{\tau} X^{A 0}(\tau) X^{0 A}\left(\tau^{\prime}\right)\right\rangle .
\end{aligned}
$$

Then, using (23) we obtain the differentiation properties in the 22 subspace (corresponding to itinerant particles):

$$
\begin{gathered}
\left(\hat{A}^{\prime}\right)_{22}\left(\hat{A}_{0}\right)_{22}=- \\
\left(\hat{A}_{0}\right)_{22}-\left(\hat{A}_{0}\right)_{22}^{2}, \quad\left(\hat{A}^{\prime}\right)_{22}\left(\hat{A}_{0}^{-1}\right)_{22}=\left(\hat{A}_{0}^{-1}\right)_{22}+1, \\
\left(\hat{A}^{\prime}\right)_{22} \hat{G}_{22}=-\left(1+\left(\hat{A}_{0}\right)_{22}\right) \hat{G}_{22} .
\end{gathered}
$$


This makes possible to write the iteration series for $\hat{L}_{22}$ as a sum of geometric progression:

$$
\begin{aligned}
\hat{L}_{22} & =\left[1+\left(\hat{A}_{0}^{-1}\right)_{22}\right]\left[J_{A}\left(\omega_{n}\right) G_{A}\left(\omega_{n}\right)-\left[J_{A}\left(\omega_{n}\right) G_{A}\left(\omega_{n}\right)\right]^{2}+\cdots\right] \\
& =-\frac{1-\left\langle X^{00}+X^{A A}\right\rangle}{\left\langle X^{00}+X^{A A}\right\rangle} \frac{J_{A}\left(\omega_{n}\right) G_{A}\left(\omega_{n}\right)}{1+J_{A}\left(\omega_{n}\right) G_{A}\left(\omega_{n}\right)} .
\end{aligned}
$$

Finally, we get the expression for the Green's function irreducible part for itinerant particles

$$
\Xi_{A}^{-1}=\frac{g_{A 0}^{-1}\left(\omega_{n}\right)-J_{A}\left(\omega_{n}\right)}{\left\langle X^{00}+X^{A A}\right\rangle}+J_{A}\left(\omega_{n}\right)
$$

This expression corresponds to the exact result (see for example [37-39]); it is also obtained within the different-time decoupling approach $[9,13,14]$.

\section{Relation of the generating functional approach to other approximations}

Let us compare the results of the generating functional approach with other approximate methods of calculating the Green's function of the single-site problem. We consider, in detail, the first iteration in the series for the total irreducible part. When the fluctuating field tends to zero $\left(v_{\alpha} \rightarrow 0\right)$, the expression for $\left(\hat{\Xi}^{-1}\right)_{11}$, as it follows from $(46)$, is

$$
\begin{aligned}
& \left(\hat{\Xi}^{-1}\right)_{11}\left(\tau-\tau^{\prime}\right)=-\frac{1}{D(\tau)} \frac{\delta \Phi_{V}}{\delta v_{0 A}(\tau)} g_{B 0}^{-1}\left(\tau-\tau^{\prime}\right) \\
& +\int_{0}^{\beta} \mathrm{d} \tau_{2} \int_{0}^{\beta} \mathrm{d} \tau_{4} \hat{J}_{22}\left(\tau-\tau_{4}\right) \hat{G}_{22}\left(\tau_{4}-\tau_{2}\right) \frac{\delta \Phi_{V}}{\delta v_{0 A}(\tau)} \frac{\delta^{2} \Phi_{V}}{\delta v(\tau) \delta \bar{v}\left(\tau_{2}\right)} \frac{g_{B 0}^{-1}\left(\tau_{2}-\tau^{\prime}\right)}{D(\tau) D\left(\tau_{2}\right)} \\
& +\int_{0}^{\beta} \mathrm{d} \tau_{4} \hat{J}_{22}\left(\tau-\tau_{4}\right) \hat{G}_{22}\left(\tau_{4}-\tau^{\prime}\right) \frac{\delta \Phi_{V}}{\delta v_{0 A}(\tau)} \frac{\delta \Phi_{V}}{\delta v_{0 B}\left(\tau^{\prime}\right)} \frac{\delta\left(\tau-\tau^{\prime}\right)}{D(\tau) D\left(\tau^{\prime}\right)} \\
& +\int_{0}^{\beta} \mathrm{d} \tau_{2} \int_{0}^{\beta} \mathrm{d} \tau_{4} \hat{J}_{11}\left(\tau-\tau_{2}\right) \hat{G}_{11}\left(\tau_{2}-\tau_{4}\right) \frac{\delta \Phi_{V}}{\delta v_{0 A}(\tau)} \frac{\delta^{2} \Phi_{V}}{\delta v_{0 B}(\tau) \delta v_{0 B}\left(\tau_{4}\right)}\left[\frac{\delta \Phi_{V}}{\delta v_{0 B}\left(\tau_{4}\right)}\right]^{-2} \frac{g_{B 0}^{-1}\left(\tau_{4}-\tau^{\prime}\right)}{D(\tau)} .
\end{aligned}
$$

Here

$$
\frac{\delta^{2} \Phi_{V}}{\delta v(\tau) \delta \bar{v}\left(\tau_{2}\right)}=G^{A B}\left(\tau-\tau_{2}\right) \equiv\left\langle\mathcal{I}_{\tau} X^{A B}(\tau) X^{B A}\left(\tau_{2}\right)\right\rangle,
$$

while the second derivative of the generating functional with respect to the field $v_{0 B}$ can be represented as a cumulant Green's function:

$$
\begin{aligned}
G_{\mathrm{c}}^{0 B}\left(\tau-\tau_{4}\right) & \left.\equiv \frac{\delta^{2} \Phi_{V}}{\delta v_{0 B}(\tau) \delta v_{0 B}\left(\tau_{4}\right)}\right|_{v=0} \\
& =\left\langle\mathcal{I}_{\tau}\left(X^{00}+X^{B B}\right)_{\tau}\left(X^{00}+X^{B B}\right)_{\tau_{4}}\right\rangle-\left\langle\mathcal{I}_{\tau}\left(X^{00}+X^{B B}\right)_{\tau}\right\rangle\left\langle\mathcal{T}_{\tau}\left(X^{00}+X^{B B}\right)_{\tau_{4}}\right\rangle .
\end{aligned}
$$

In the frequency representation

$$
\left(\hat{\Xi}^{-1}\right)_{11}\left(\omega_{n}\right)=\frac{1}{A_{0 B}}\left\{g_{B 0}^{-1}\left(\omega_{n}\right)\left[1-Q_{B}\left(\omega_{n}\right)-N_{B}\left(\omega_{n}\right)\right]+\frac{1}{A_{0 A}} \tilde{S}_{B}\right\},
$$

where

$$
Q_{B}\left(\omega_{n}\right)=\left(\frac{\delta \Phi_{V}}{\delta v_{0 A}}\right)^{-2} \frac{1}{\beta} \sum_{\omega_{m}} \mathrm{e}^{\mathrm{i} \omega_{m} 0^{+}} J_{B}\left(\omega_{m}\right) G_{\mathrm{c}}^{0 B}\left(\omega_{m}-\omega_{n}\right)
$$




$$
\begin{gathered}
N_{B}\left(\omega_{n}\right)=\frac{1}{\beta} \sum_{\omega_{m}} \mathrm{e}^{\mathrm{i} \omega_{m} 0^{+}} J_{A}\left(\omega_{m}\right) G_{A}\left(\omega_{m}\right) G^{A B}\left(\omega_{m}-\omega_{n}\right) \frac{1}{D}, \\
\tilde{S}_{B}=\frac{1}{\beta} \sum_{\omega_{m}} \mathrm{e}^{\mathrm{i} \omega_{m} 0^{+}} J_{A}\left(\omega_{m}\right) G_{A}\left(\omega_{m}\right) .
\end{gathered}
$$

Formula (81) gives a full expression for the inverse irreducible part $\hat{\Xi}^{-1}$ in the linear, with respect to $J_{\sigma}$, approximation.

\subsection{Comparison with $\mathrm{GH} 3$}

Basing on the obtained expression for $\hat{\Xi}^{-1}$, let us consider some of its possible simplifications. At first, we compare the result (81) of the generating functional approach with the irreducible part $\Xi^{-1}$ obtained in the GH3 approximation [9]. For this purpose we consider the fermionic and bosonic Green's functions in (81) in the zero approximation:

$$
\begin{gathered}
G^{A B}\left(\omega_{m}\right) \approx\left\langle X^{A A}-X^{B B}\right\rangle g_{A B}\left(\omega_{m}\right), \quad G_{\mathrm{c}}^{0 B}\left(\omega_{m}\right) \approx \beta \delta\left(\omega_{m}\right) A_{0 B}\left(1-A_{0 B}\right), \\
G_{A}\left(\omega_{m}\right) \approx A_{0 A} g_{A 0}\left(\omega_{m}\right), \quad G_{B}\left(\omega_{m}\right) \approx A_{0 B} g_{B 0}\left(\omega_{m}\right) \\
g_{p q}=\frac{1}{\mathrm{i} \omega-\lambda_{p q}}, \quad \lambda_{p q}=\lambda_{p}-\lambda_{q}
\end{gathered}
$$

where $\lambda_{p(q)}$ are eigenvalues of the unperturbed singe-site Hamiltonian. This approximation allows us to apply the following identity

$$
\frac{1}{\mathrm{i} \omega_{m}-\lambda_{A 0}} \frac{1}{\mathrm{i} \omega_{m}-\mathrm{i} \omega_{n}-\lambda_{A B}}=\frac{-1}{\mathrm{i} \omega_{n}-\lambda_{B 0}}\left[\frac{1}{\mathrm{i} \omega_{m}-\lambda_{A 0}}-\frac{1}{\mathrm{i} \omega_{m}-\mathrm{i} \omega_{n}-\lambda_{A B}}\right]
$$

calculating the product $g_{B 0}^{-1}\left(\omega_{n}\right) N_{B}\left(\omega_{n}\right)$ in (81). Thus, we obtain

$$
Q_{B}\left(\omega_{n}\right)=\left(1-A_{0 B}\right) J_{B}\left(\omega_{n}\right) g_{B 0}\left(\omega_{n}\right)
$$

and

$$
\left(\Xi^{-1}\right)_{11}\left(\omega_{n}\right)=\frac{1}{A_{0 B}}\left[g_{0 B}^{-1}\left(\omega_{n}\right)-\frac{1}{A_{0 B}} P_{B}\left(\omega_{n}\right)+\frac{1}{A_{0 B}} \tilde{S}_{B}\left(\omega_{n}\right)-\left(1-A_{0 B}\right) J_{B}\left(\omega_{n}\right)\right]
$$

where

$$
P_{B}\left(\omega_{n}\right)=\frac{1}{\beta} \sum_{\omega_{m}} \mathrm{e}^{\mathrm{i} \omega_{m} 0^{+}} J_{A}\left(\omega_{m}\right) \frac{\left\langle X^{A A}-X^{B B}\right\rangle}{\mathrm{i} \omega_{m}-\mathrm{i} \omega_{n}-\lambda_{A B}}
$$

is the function that in diagram representation corresponds to the loop containing the lines of the coherent potential $J_{A}$ and unperturbed bosonic Green's function $g_{A B}$ oriented in the opposite directions. On the other hand, such a loop appears from different-time decoupling of the Green's function $\left\langle\left\langle X^{A B} \xi_{A} \mid \xi_{A}^{\dagger} X^{B A}\right\rangle\right\rangle_{\omega}$ that is contributing to the total irreducible part of the single-site Green's function in the equation of motion approach in the GH3 approximation $[9,14]$.

The coherent potential $J_{\sigma}$ can be written in the Lehmann representation

$$
J_{\sigma}\left(\omega_{n}\right)=-\frac{1}{\pi} \lim _{\eta \rightarrow 0^{+}} \int_{-\infty}^{+\infty} \frac{\operatorname{Im} J_{\sigma}\left(\omega^{\prime}+\mathrm{i} \eta\right) \mathrm{d} \omega^{\prime}}{\mathrm{i} \omega_{n}-\omega^{\prime}} .
$$

Using the relation of the (88) type as well as formulae

$$
\begin{aligned}
\frac{1}{\beta} \sum_{\omega_{m}} \frac{\left\langle X^{A A}+X^{B B}\right\rangle}{\mathrm{i} \omega_{m}-\mathrm{i} \omega_{n}-\lambda_{A B}} \mathrm{e}^{\mathrm{i} \omega_{m} 0^{+}} & =\left\langle X^{A A}\right\rangle \\
\frac{1}{\beta} \sum_{\omega_{m}} \frac{1}{\mathrm{i} \omega_{m}-\omega^{\prime}} \mathrm{e}^{\mathrm{i} \omega_{m} 0^{+}} & =\frac{1}{\mathrm{e}^{\beta \omega^{\prime}}+1}=\frac{1}{2}-\frac{1}{2} \tanh \frac{\beta \omega^{\prime}}{2}
\end{aligned}
$$


we obtain the expression for $P_{B}$ in the form of integral over frequency.

$$
\begin{aligned}
& P_{B}\left(\omega_{n}\right)=\frac{\left\langle X^{A A}+X^{B B}\right\rangle}{2} J_{A}\left(\mathrm{i} \omega_{n}+\lambda_{A B}\right)+\frac{\left\langle X^{A A}-X^{B B}\right\rangle}{2} Y_{B}\left(\omega_{n}\right), \\
& Y_{B}\left(\omega_{n}\right)=\frac{1}{2 \pi} \int_{-\infty}^{+\infty} \frac{\left[-2 \operatorname{Im} J_{A}\left(\omega^{\prime}+\mathrm{i} 0^{+}\right)\right]}{\mathrm{i} \omega_{n}-\omega^{\prime}+\lambda_{A B}} \tanh \frac{\beta \omega^{\prime}}{2} \mathrm{~d} \omega^{\prime} .
\end{aligned}
$$

Considering the expression for the Green's function

$$
G_{B}\left(\omega_{n}\right)=\frac{A_{0 B}}{g_{B 0}^{-1}\left(\omega_{n}\right)-\frac{P_{B}\left(\omega_{n}\right)}{A_{0 B}}+\frac{\tilde{S}_{B}}{A_{0 B}}-J_{B}\left(\omega_{n}\right)}
$$

one can see that (96) together with (95) corresponds to the GH3 approximation. The correspondence: $P_{B}\left(\omega_{n}\right) \leftrightarrow-R_{B}(\omega) ; \tilde{S}_{B} \leftrightarrow-V_{A} \varphi_{B}$ (in the notations used in [9]) takes place.

\subsection{Extension of JK decoupling to AHM}

Our next step is to compare the obtained expression (81) for $\hat{\Xi}^{-1}$ with the expression that can be found for the single-site problem of AHM within the framework of the equation of motion approach using the decoupling [40] used by Jeschke and Kotliar in the paper [23]. Their scheme for the model with the degeneracy and with the exclusion of the doubly occupied states can be easily extended to the case when $\mu_{A} \neq \mu_{B}$. Following the scheme proposed in [23] for solving the DMFT problem, we obtain (see Appendix)

$$
G_{\sigma}=\frac{A_{0 \sigma}+I_{1, \bar{\sigma}}(\omega)}{\omega-\lambda_{\sigma 0}+J_{\sigma}(\omega)+I_{2, \bar{\sigma}}(\omega)-J_{\sigma}(\omega) I_{1, \bar{\sigma}}(\omega)} .
$$

Let us write the corresponding expression for the irreducible part and linearize it with respect to the coherent potential $\left(J_{\sigma}\right)$ :

$$
\begin{aligned}
\Xi_{\sigma}^{-1}\left(\omega_{n}\right) & =\frac{g_{\sigma 0}^{-1}\left(\omega_{n}\right)+I_{2, \bar{\sigma}}\left(\omega_{n}\right)-J_{\sigma}\left(\omega_{n}\right) I_{1, \bar{\sigma}}\left(\omega_{n}\right)-J_{\sigma}\left(\omega_{n}\right)}{A_{0 \sigma}+I_{1, \bar{\sigma}}\left(\omega_{n}\right)}+J_{\sigma} \\
& \approx \frac{1}{A_{0 \sigma}}\left[g_{\sigma 0}^{-1}\left(\omega_{n}\right)-\left(1-A_{0 \sigma}\right) J_{\sigma}\left(\omega_{n}\right)+I_{2, \bar{\sigma}}\left(\omega_{n}\right)-g_{\sigma 0}^{-1} \frac{I_{1, \bar{\sigma}}\left(\omega_{n}\right)}{A_{0 \sigma}}\right] .
\end{aligned}
$$

We transform the expressions for functions $I_{1, \bar{\sigma}}(111)$ and $I_{2, \bar{\sigma}}$ (112) using the zero approximation for electron Green's function $\left(\left\langle\left\langle X^{0 A} \mid X^{A 0}\right\rangle\right\rangle_{\mathrm{i} \omega_{m}}=A_{0}\left[\mathrm{i} \omega_{m}-\lambda_{A 0}\right]^{-1}\right)$ and the scheme described in the previous subsection. As a result we obtain:

$$
\begin{gathered}
g_{B 0}^{-1}\left(\omega_{n}\right) I_{1, A}\left(\omega_{n}\right)=-\frac{\left\langle X^{A A}-X^{00}\right\rangle}{2} J_{A}\left(\mathrm{i} \omega_{n}+\lambda_{A B}\right)+\frac{\left\langle X^{A A}+X^{00}\right\rangle}{2} Y_{B}\left(\omega_{n}\right)-\tilde{S}_{B}, \\
I_{2, A}\left(\omega_{n}\right)=-\frac{1}{2} J_{A}\left(\mathrm{i} \omega_{n}+\lambda_{A B}\right)+\frac{1}{2} Y_{B}\left(\omega_{n}\right) .
\end{gathered}
$$

In its turn, it leads to the relation

$$
I_{2, A}\left(\omega_{n}\right)-g_{B 0}^{-1} \frac{I_{1, A}\left(\omega_{n}\right)}{A_{0 B}}=\frac{\tilde{S}_{B}}{A_{0 B}}-\frac{P_{B}\left(\omega_{n}\right)}{A_{0 B}} .
$$

One can see that in this case expressions (98) and (96) coincide.

Therefore, the JK decoupling scheme corresponds to the GH3 approximation after the replacement $G_{\sigma} \rightarrow G_{\sigma}^{0}$ and at the linearization of $\Xi_{\sigma}^{-1}$ with respect to $J_{\sigma,(\bar{\sigma})}$. Besides, such a scheme corresponds to the linear approximation in the generating functional approach, while in the latter the fermionic and bosonic Green's functions are considered in the zero approximation. 


\section{Conclusions}

The generating functional approach developed based on the Kadanoff-Baym idea by Izyumov et al. is adapted to solving the single-site problem in the dynamical mean field theory. To construct the equations for Green's functions the recurrent form of Wick's theorem for Hubbard operators is used. This approach is similar to the usual scheme where the equations are constructed using differentiation with respect to the time variable. However, when there are more than one operators with the same time that is differentiated, these methods are different.

Within the framework of the generating functional approach a closed self-consistent equation in functional derivatives is obtained for constructing the irreducible part (self energy, Green's function, etc.). To solve this equation the iterative procedure is used. It is shown that the solution for the irreducible part can be expressed in terms of two functions. These functions are formed by the different types of terms in the iteration series: one function involves the derivatives of linear terms of fluctuating fields which are present in the $g^{-1}$ function; the other one can be considered as a terminating part of the Green's function (or the irreducible part). As an example, the asymmetric Hubbard model is considered.

The algorithm is given for constructing the inverse irreducible part $\Xi_{\sigma}^{-1}, \sigma=\{A, B\}$ (or the mass operator $F_{\sigma}$ and terminating part $\Lambda_{\sigma}$ ) in the form of a series in powers of coherent potentials $J_{A}, J_{B}$. The irreducible part in the approximation linear with respect to $J_{\sigma}$ is obtained and analysed explicitly. Comparison is performed with the results of other approximations based on the decoupling of the single-site Hubbard operator Green's functions of higher order in equations of motion. It is established that after replacing fermionic and bosonic Green's functions in the expression for $\Xi_{\sigma}^{-1}$ by their zero approximations, this expression is reduced to the corresponding expression in the GH3 approximation. Besides, at the similar simplification, the decoupling scheme used by Jeschke and Kotliar for the Hubbard-like models can be also reduced to the GH3 approximation. It should be mentioned, that such a scheme uses the zero-order bosonic Green's functions. Therefore it is also more simple than the generating functional approach formulated in the approximation linear with respect to $J_{\sigma}$.

It should be noted that, in the proposed approach, a full closure of equations for the Green's function requires calculation of bosonic Green's functions appearing in the expression for $\Xi^{-1}$ (see for example (81)). It also can be done within the framework of the generating functional approach, similarly as it was done in [35] using equations of motion with functional derivatives.

\section{A. Decoupling for Anderson impurity model for AHM}

In the equation of motion decoupling method proposed by Jeschke and Kotliar [23], the DMFT single-site problem is interpreted as Anderson impurity model. In this case, when $U \rightarrow \infty$, the Hamiltonian of the model reads

$$
\hat{H}=\sum_{k \sigma} \varepsilon_{k \sigma} c_{k \sigma}^{\dagger} c_{k \sigma}+\sum_{\sigma} E_{\sigma} X^{\sigma \sigma}+E_{0} X^{00}+\sum_{k \sigma}\left(V_{k \sigma}^{*} c_{k \sigma}^{\dagger} X^{0 \sigma}+V_{k \sigma} X^{\sigma 0} c_{k \sigma}\right),
$$

where $\sigma=A, B ; V_{k \sigma}$ is the hybridization parameter. Unlike the similar problem for the standard Hubbard model, here $E_{A} \neq E_{B}, \varepsilon_{k A} \neq \varepsilon_{k B}, V_{k A} \neq V_{k B}$.

To determine the Green's function $\left\langle\left\langle X^{0 \sigma} \mid X^{\sigma 0}\right\rangle\right\rangle$ the equation of motion approach is used. At the first stage, the Green's functions $\left\langle\left\langle\left(X^{00}+X^{\sigma \sigma}\right) c_{k \sigma} \mid X^{\sigma 0}\right\rangle\right\rangle$ and $\left\langle\left\langle X^{\bar{\sigma} \sigma} c_{k \bar{\sigma}} \mid X^{\sigma 0}\right\rangle\right\rangle$ appear; they are calculated using the equations of motion and differentiating again the left time argument. The Green's functions of higher order appearing at the second stage are decoupled [23]. This procedure leads to the average values $\left\langle c_{k \bar{\sigma}} X^{\bar{\sigma} 0}\right\rangle$ and $\left\langle c_{k^{\prime} \bar{\sigma}}^{\dagger} c_{k \bar{\sigma}}\right\rangle$ that are determined self-consistently.

As a result, the initial function $\left\langle\left\langle X^{0 \sigma} \mid X^{\sigma 0}\right\rangle\right\rangle$ is determined from the set of equations

$$
\begin{aligned}
& \left(\omega-\lambda_{\sigma 0}\right)\left\langle\left\langle X^{0 \sigma} \mid X^{\sigma 0}\right\rangle\right\rangle_{\omega}=A_{0 \sigma}+\Delta_{\sigma}(\omega)\left\langle\left\langle X^{0 \sigma} \mid X^{\sigma 0}\right\rangle\right\rangle_{\omega} \\
& +I_{1, \bar{\sigma}}(\omega)-I_{2, \bar{\sigma}}(\omega)\left\langle\left\langle X^{0 \sigma} \mid X^{\sigma 0}\right\rangle\right\rangle_{\omega}+I_{1, \bar{\sigma}}(\omega) \sum_{k^{\prime}} V_{k^{\prime} \sigma}\left\langle\left\langle c_{k^{\prime} \sigma} \mid X^{\sigma 0}\right\rangle\right\rangle_{\omega}, \\
& \left(\omega-\varepsilon_{k^{\prime} \sigma}\right)\left\langle\left\langle c_{k^{\prime} \sigma} \mid X^{\sigma 0}\right\rangle\right\rangle_{\omega}=V_{k^{\prime} \sigma}^{*}\left\langle\left\langle X^{0 \sigma} \mid X^{\sigma 0}\right\rangle\right\rangle_{\omega},
\end{aligned}
$$


where

$$
\begin{gathered}
\lambda_{\sigma \bar{\sigma}}=E_{\sigma}-E_{\bar{\sigma}} ; \quad \lambda_{\sigma 0}=E_{\sigma}-E_{0} ; \quad A_{0 \sigma}=\left\langle X^{00}+X^{\sigma \sigma}\right\rangle, \\
\Delta_{\sigma}(\omega)=\sum_{k} \frac{\left|V_{k \sigma}\right|^{2}}{\omega-\varepsilon_{k \sigma}}, \\
I_{1, \bar{\sigma}}(\omega)=\sum_{k} V_{k \bar{\sigma}} \frac{\left\langle c_{k \bar{\sigma}} X^{\bar{\sigma} 0}\right\rangle}{\omega-\lambda_{\sigma \bar{\sigma}}-\varepsilon_{k \bar{\sigma}}} ; \quad I_{2, \bar{\sigma}}(\omega)=-\sum_{k k^{\prime}} V_{k \bar{\sigma}} V_{k^{\prime} \bar{\sigma}}^{*} \frac{\left\langle c_{k \bar{\sigma}}^{\dagger} c_{k \bar{\sigma}}\right\rangle}{\omega-\lambda_{\sigma \bar{\sigma}}-\varepsilon_{k \bar{\sigma}}} .
\end{gathered}
$$

As a result we come to

$$
G_{\sigma}=\frac{A_{0 \sigma}+I_{1, \bar{\sigma}}(\omega)}{\omega-\lambda_{\sigma 0}+\Delta_{\sigma}(\omega)+I_{2, \bar{\sigma}}(\omega)-\Delta_{\sigma}(\omega) I_{1, \bar{\sigma}}(\omega)} .
$$

In this approach, the hybridization function $\Delta_{\sigma}(\omega)$ corresponds to the coherent potential: $\Delta_{\sigma}(\omega)=J_{\sigma}(\omega)$ (see [23] and references therein).

To calculate the average values in (107), the following method is used

$$
\begin{aligned}
\left\langle c_{k \bar{\sigma}} X^{\bar{\sigma} 0}\right\rangle & =-\frac{1}{\beta} \sum_{\omega_{n}}\left\langle\left\langle c_{k \bar{\sigma}} \mid X^{\bar{\sigma} 0}\right\rangle\right\rangle_{\mathrm{i} \omega_{n}} \mathrm{e}^{\mathrm{i} \omega_{n} 0^{+}} \\
\left\langle c_{k^{\prime} \bar{\sigma}}^{\dagger} c_{k \bar{\sigma}}\right\rangle & =\frac{1}{\beta} \sum_{\omega_{n}}\left\langle\left\langle c_{k \bar{\sigma}} \mid c_{k^{\prime} \bar{\sigma}}^{\dagger}\right\rangle\right\rangle_{\mathrm{i} \omega_{n}} \mathrm{e}^{\mathrm{i} \omega_{n} 0^{+}}=\frac{1}{\beta} \sum_{\omega_{n}}\left[\frac{\delta_{k k^{\prime}}}{\mathrm{i} \omega_{n}-\varepsilon_{k \bar{\sigma}}}+\frac{V_{k \bar{\sigma}}^{*} V_{k^{\prime} \bar{\sigma}}}{\left(\mathrm{i} \omega_{n}-\varepsilon_{k \bar{\sigma}}\right)\left(\mathrm{i} \omega_{n}-\varepsilon_{\left.k^{\prime} \bar{\sigma}\right)}\right)}\right] .
\end{aligned}
$$

Following the procedure described in [23], we obtain

$$
\begin{aligned}
I_{1, \bar{\sigma}}\left(\mathrm{i} \omega_{n}\right)= & -\frac{1}{\beta} \sum_{\omega_{n}} \mathrm{e}^{\mathrm{i} \omega_{m} 0^{+}} \Delta_{\bar{\sigma}}\left(\mathrm{i} \omega_{m}\right) \frac{1}{\mathrm{i} \omega_{n}-\mathrm{i} \omega_{m}-\lambda_{\sigma \bar{\sigma}}}\left\langle\left\langle X^{0 \bar{\sigma}} \mid X^{\bar{\sigma} 0}\right\rangle\right\rangle_{\mathrm{i} \omega_{m}} \\
& +\frac{1}{\beta} \sum_{\omega_{n}} \mathrm{e}^{\mathrm{i} \omega_{m} 0^{+}} \Delta_{\bar{\sigma}}\left(\mathrm{i} \omega_{n}-\lambda_{\sigma \bar{\sigma}}\right) \frac{1}{\mathrm{i} \omega_{n}-\mathrm{i} \omega_{m}-\lambda_{\sigma \bar{\sigma}}}\left\langle\left\langle X^{0 \bar{\sigma}} \mid X^{\bar{\sigma} 0}\right\rangle\right\rangle_{\mathrm{i} \omega_{m}}, \\
I_{2, \bar{\sigma}}\left(\mathrm{i} \omega_{n}\right)= & -\frac{1}{\beta} \sum_{\omega_{n}} \mathrm{e}^{\mathrm{i} \omega_{m} 0^{+}}\left[\Delta_{\bar{\sigma}}\left(\mathrm{i} \omega_{m}\right)-\Delta_{\bar{\sigma}}\left(\mathrm{i} \omega_{n}-\lambda_{\sigma \bar{\sigma}}\right)\right] \frac{1}{\mathrm{i} \omega_{n}-\mathrm{i} \omega_{m}-\lambda_{\sigma \bar{\sigma}}} \\
& -\frac{1}{\beta} \sum_{\omega_{n}} \mathrm{e}^{\mathrm{i} \omega_{m} 0^{+}} \frac{\left[\Delta_{\bar{\sigma}}\left(\mathrm{i} \omega_{m}\right)-\Delta_{\bar{\sigma}}\left(\mathrm{i} \omega_{n}-\lambda_{\sigma \bar{\sigma}}\right)\right]}{\mathrm{i} \omega_{n}-\mathrm{i} \omega_{m}-\lambda_{\sigma \bar{\sigma}}}\left\langle\left\langle X^{0 \bar{\sigma}} \mid X^{\bar{\sigma} 0}\right\rangle\right\rangle_{\mathrm{i} \omega_{m}} \Delta_{\bar{\sigma}}\left(\mathrm{i} \omega_{m}\right) .
\end{aligned}
$$

In comparison with [23], where the case of degeneracy $\left(E_{\sigma}=-\mu\right)$ was considered, in the expressions (111) and (112), instead of the factor $\left[i \omega_{n}-\mathrm{i} \omega_{m}\right]^{-1}$, the unperturbed bosonic Green's function $\left[\mathrm{i} \omega_{n}-\mathrm{i} \omega_{m}-\lambda_{\sigma \bar{\sigma}}\right]^{-1}$ is present.

\section{References}

1. Izyumov Y.A., UFN, 1995, 165, 403. [Izyumov Y.A., Phys. Usp., 1995, 38, 385].

2. Georges A., Kotliar G., Krauth W., Rozenberg M.J., Rev. Mod. Phys., 1996, 68, 13.

3. Freericks J.K., Zlatić V., Rev. Mod. Phys., 2003, 75, 1333.

4. Kocharian A.N., Reich G.R., J. Appl. Phys., 1994, 76, 6127.

5. Ueltschi D., J. Stat. Phys., 2004, 116, 681.

6. Batista C.D., Phys. Rev. Lett., 2002, 89, 166403.

7. Fáth G., Domański Z., Lemański R., Phys. Rev. B, 1995, 52, 13910.

8. Macedo C.A., de Souza A.M.C., Phys. Rev. B, 2002, 65, 153109.

9. Stasyuk I.V., Hera O.B., Phys. Rev. B, 2005, 72, 045134.

10. Stasyuk I.V., Hera O.B., Eur. Phys. J. B, 2005, 48, 439.

11. Metzner W., Vollhardt D., Phys. Rev. Lett., 1989, 62, 324.

12. Georges A., Kotliar G., Phys. Rev. B, 1992, 45, 6479.

13. Stasyuk I.V., Condens. Matter Phys., 2000, 3, 437.

14. Stasyuk I.V., Hera O.B., Condens. Matter Phys., 2003, 6, 127. 
15. Jarrell M., Phys. Rev. Lett., 1992, 69, 168.

16. Rozenberg M.J., Zhang X.Y., Kotliar G., Phys. Rev. Lett., 1992, 69, 1236.

17. Georges A., Krauth W., Phys. Rev. Lett., 1992, 69, 1240.

18. Caffarel M., Krauth W., Phys. Rev. Lett., 1994, 72, 1545.

19. Si Q., Rozenberg M.J., Kotliar G., Ruckenstein A.E., Phys. Rev. Lett., 1994, 72, 2761.

20. Bulla R., Adv. Solid State Phys., 2000, 40, 169.

21. Held K., Nekrasov I.A., Keller G., Eyert V., Blümer N., McMahan A.K., Scalettar R.T., Pruschke T., Anisimov V.I., Vollhardt D., Psi-k Newsletter, 2003, 56, 65.

22. Georges A., AIP Conference Proceedings, 2004, 715, 3.

23. Jeschke H.O., Kotliar G., Phys. Rev. B, 2005, 71, 085103.

24. Dai X., Haule K., Kotliar G., Phys. Rev. B, 2005, 72, 045111.

25. Shvaika A.M., Phys. Rev. B, 2000, 62, 2358.

26. Bickers N.E., Cox D.L., Wilkins J.W., Phys. Rev. B, 1987, 36, 2036.

27. Zölfl M.B., Pruschke T., Keller J., Poteryaev A.I., Nekrasov I.A., Anisimov V.I., Phys. Rev. B, 2000, 61, 12810.

28. Zhang X.Y., Rozenberg M.J., Kotliar G., Phys. Rev. Lett., 1993, 70, 1666.

29. Potthoff M., Herrmann T., Wegner T., Nolting W., phys. stat. sol. (b), 1998, 210, 199.

30. Hubbard J., Proc. R. Soc. London, Ser. A, 1964, 281, 401.

31. Kadanoff L.P., Baym G., Quantum statistical mechanics, W.A. Benjamin, Inc., New York, 1962.

32. Izyumov Y.A., Chaschin N.I., The Physics of Metals and Metallography, 2001, 92, 451.

33. Izyumov Y.A., Chaschin N.I., The Physics of Metals and Metallography, 2001, 92, 351.

34. Izyumov Y.A., Chaschin N.I., Yushankhai V.Y., Phys. Rev. B, 2002, 65, 214425.

35. Izyumov Y.A., Chaschin N.I., Alexeev D.S., Mancini F., Eur. Phys. J. B, 2005, 45, 69.

36. Slobodyan P.M., Stasyuk I.V., Teor. Mat. Fiz., 1974, 19, 423. [ Slobodyan P.M., Stasyuk I.V., Theor. Math. Phys. USSR, 1974, 19,616].

37. Brandt U., Mielsch C., Z. Phys. B, 1989, 75, 365.

38. Brandt U., Mielsch C., Z. Phys. B, 1990, 79, 295.

39. Brandt U., Mielsch C., Z. Phys. B, 1991, 82, 37.

40. Luo H.G., Ying J.J., Wang S.J., Phys. Rev. B, 1999, 59, 9710.

\title{
Асиметрична модель Хаббарда в теорії динамічного середнього поля в методі твірного функціоналу
}

\author{
І.В.Стасюк, О.Б.Гера \\ Інститут фізики конденсованих систем НАН України, 79011 Львів, вул. Свєнціцького, 1
}

Отримано 6 червня 2006 р.

В роботі розвивається новий аналітичний підхід для розв'язання ефективної одновузлової задачі в методі динамічного середнього поля. Підхід ґрунтується на методі твірного функціоналу КадановаБейма у формі, розробленій в роботах Ізюмова та ін. Він дає можливість отримати замкнене рівняння у функціональних похідних для незвідної частини одновузлової функції Гріна частинок; розв'язки будуються ітеративним способом. В ролі застосування запропонованої схеми взято асиметричну модель Хаббарда (AMX). Побудовано обернену незвідну частину $\Xi_{\sigma}^{-1}$ одновузлової функції Гріна в лінійному наближенні за когерентним потенціалом $J_{\sigma}$. Виходячи з отриманого результату, розглянено функцію Гріна рухомих частинок у границі Фалікова-Кімбала AMX, проаналізовано схеми розщеплень у рівняннях руху для одновузлової функції Гріна (наближення $\mathrm{GH} 3$, розщеплення ЄшкеКотляра).

Ключові слова: сильноскорельовані системи, асиметрична модель Хаббарда, одновузлова задача, теорія динамічного середнього поля

PACS: $71.10 . F d, 71.27 .+a, 71.30 .+h$ 